\title{
Learning Language Specific Sub-network for Multilingual Machine Translation
}

\author{
Zehui Lin, Liwei Wu, Mingxuan Wang, Lei Li \\ ByteDance AI Lab \\ \{linzehui,wuliwei.000,wangmingxuan.89,lileilab\}@bytedance.com
}

\begin{abstract}
Multilingual neural machine translation aims at learning a single translation model for multiple languages. These jointly trained models often suffer from performance degradation on rich-resource language pairs. We attribute this degeneration to parameter interference. In this paper, we propose LaSS to jointly train a single unified multilingual MT model. LaSS learns Language Specific Sub-network (LaSS) for each language pair to counter parameter interference. Comprehensive experiments on IWSLT and WMT datasets with various Transformer architectures show that LaSS obtains gains on 36 language pairs by up to 1.2 BLEU. Besides, LaSS shows its strong generalization performance at easy adaptation to new language pairs and zero-shot translation. LaSS boosts zero-shot translation with an average of 8.3 BLEU on 30 language pairs. Codes and trained models are available at https: //github.com/NLP-Playground/LaSS.
\end{abstract}

\section{Introduction}

Neural machine translation (NMT) has been very successful for bilingual machine translation (Bahdanau et al., 2015; Vaswani et al., 2017; Wu et al., 2016; Hassan et al., 2018; Su et al., 2018; Wang, 2019). Recent research has demonstrated the efficacy of multilingual NMT, which supports translation from multiple source languages into multiple target languages with a single model (Johnson et al., 2017; Aharoni et al., 2019; Zhang et al., 2020; Fan et al., 2020; Siddhant et al., 2020). Multilingual NMT enjoys the advantage of deployment. Further, the parameter sharing of multilingual NMT encourages transfer learning of different languages. An extreme case is zero-shot translation, where direct translation between a language pair never seen in training is possible (Johnson et al., 2017).

\footnotetext{
${ }^{*}$ Equal contribution.
}

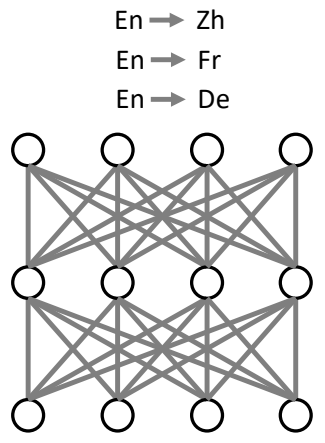

(a) Full network

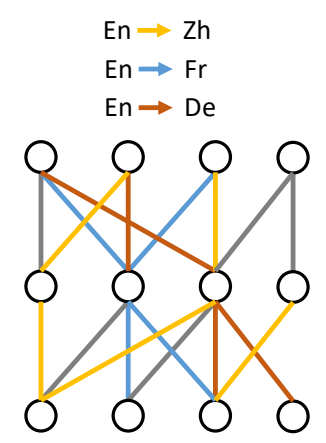

(b) LaSS
Figure 1: Illustration of a full network and languagespecific ones (LaSS). - represents shared weights. , - and - represents weights for En $\rightarrow \mathrm{Zh}, \mathrm{En} \rightarrow \mathrm{Fr}$ and $\mathrm{En} \rightarrow \mathrm{De}$, respectively. Compared to the full multilingual model, each LaSS learned model has language universal and language specific weights.

While very promising, several challenges remain in multilingual NMT. The most challenging one is related to the insufficient model capacity. Since multiple languages are accommodated in a single model, the modeling capacity of NMT model has to be split for different translation directions (Aharoni et al., 2019). Therefore, multilingual NMT models often suffer from performance degradation compared with their corresponding bilingual baseline, especially for rich-resource translation directions. The simplistic way to alleviate the insufficient model capacity is to enlarge the model parameters (Aharoni et al., 2019; Zhang et al., 2020). However, it is not parameter or computation efficient and needs larger multilingual training datasets to avoid over-fitting. An alternative solution is to design language-aware components, such as division of the hidden cells into shared and language-dependent ones (Wang et al., 2018), adaptation layers (Bapna and Firat, 2019; Philip et al., 2020), language-aware layer normalization 
and linear transformation (Zhang et al., 2020), and latent layers (Li et al., 2020).

In this work, we propose LaSS, a method to dynamically find and learn Language Specific Subnetwork for multilingual NMT. LaSS accommodates one sub-network for each language pair. Each sub-network has shared parameters with some other languages and, at the same time, preserves its language specific parameters. In this way, multilingual NMT can model language specific and language universal features for each language pair in one single model without interference. Figure 1 is the illustration of vanilla multilingual model and LaSS. Each language pair in LaSS has both language universal and language specific parameters. The network itself decides the sharing strategy.

The advantages of our proposed method are

- LaSS is parameter efficient, requiring no extra trainable parameters to model language specific features.

- LaSS alleviates parameter interference, potentially improving the model capacity and boosting performance.

- LaSS shows its strong generalization performance at easy adaptation to new language pairs and zero-shot translation. LaSS can be easily extended to new language pairs without dramatic degradation of existing language pairs. Besides, LaSS can boost zero-shot translation by up to 26.5 BLEU.

\section{Related Work}

Multilingual Neural Machine Translation The standard multilingual NMT model uses a shared encoder and a shared decoder for different languages (Johnson et al., 2017). There is a transfer-interference trade-off in this architecture (Arivazhagan et al., 2019): boosting the performance of low resource languages or maintain the performance of high resource languages. To solve this trade-off, previous works assign some parts of the model to be language specific: Language specific decoders (Dong et al., 2015), Language specific encoders and decoders (Firat et al., 2016; Lyu et al., 2020) and Language specific hidden states and embeds (Wang et al., 2018). Sachan and Neubig (2018) compares different sharing methods and finds different sharing methods have a great impact on performance. Recently, Zhang et al. (2021) analyze when and where language specific capacity matters. Li et al.
(2020) uses a binary conditional latent variable to decide which language each layer belongs to.

Model Pruning Our approach follows the standard pattern of model pruning: training, finding the sparse network and fine-tuning (Frankle and Carbin, 2019; Liu et al., 2019). Frankle and Carbin (2019) and Liu et al. (2019) highlight the importance of the sparse network architecture. Zhu and Gupta (2018) proposed a method to automatically adjust the sparse threshold. Sun et al. (2020) learns different sparse architecture for different tasks. Evci et al. (2020) iteratively redistribute the sparse network architecture by the gradient.

\section{Methodology}

We describe LaSS method in this section. The goal is to learn a single unified model for many translation directions. Our overall idea is to find sub-networks corresponding to each language pair, and then only update the parameters of those subnetworks during the joint training.

\subsection{Multilingual NMT}

A multilingual NMT model learns a mapping function $f$ from a sentence in one of many languages to another language. We adopt the multilingual Transformer (mTransformer) as the backbone network (Johnson et al., 2017). mTransformer has the same encoder-decoder architecture with layers of multihead attention, residual connection, and layer normalization. In addition, it has two lanuage identifying tokens for the source and target. Define a multilingual dataset $\left\{\mathcal{D}_{s_{i} \rightarrow t_{i}}\right\}_{i=1}^{N}$ where $s_{i}, t_{i}$ represents the source and target language.

We train an initial multilingual MT model with the following loss.

$$
\mathcal{L}=\sum_{i} \sum_{\langle\mathbf{x}, \mathbf{y}\rangle \sim \mathcal{D}_{s_{i} \rightarrow t_{i}}}-\log P_{\theta}(\mathbf{y} \mid \mathbf{x})
$$

where $\langle\mathbf{x}, \mathbf{y}\rangle$ is a sentence pair from the language $s_{i}$ to $t_{i}$, and $\theta$ is the model parameter.

\subsection{Finding Language Specific Model Masks}

Training a single model jointly on multiple language directions will lead to performance degradation for rich resource pairs (Johnson et al., 2017). The single model will improve on low resource language pairs, but will reduce performance on pairs like English-German. Intuitively, jointly training on all translation pairs will obtain an "average" 
model. For rich resources, such averaging may hurt the performance since a multilingual MT model must distribute its modeling capacity for all translation directions. Based on this intuition, our idea is to find a sub-network of the original multilingual model. Such sub-network is specific to each language pair.

We start from a multilingual base model $\theta_{0}$. The $\theta_{0}$ is trained with Eq. (1). A sub-network is indicated by a binary mask vector $\mathbf{M}_{s_{i} \rightarrow t_{i}} \in\{0,1\}^{|\theta|}$ for language pair $s_{i} \rightarrow t_{i}$. Each element being 1 indicates to retain the weight and 0 to abandon the weight. Then the parameters associated with $s_{i} \rightarrow t_{i}$ is $\theta_{s_{i} \rightarrow t_{i}}=\left\{\theta_{0}^{j} \mid \mathbf{M}_{s_{i} \rightarrow t_{i}}^{j}=1\right\}$, where $j$ denotes the $j$ th element in $\theta_{0}$. The parameters $\theta_{s_{i} \rightarrow t_{i}}$ are only responsible for the particular language $s_{i}$ and $t_{i}$. We intend to find such language specific sub-networks. Figure 1 illustrates the original model and its language specific sub-networks.

Given an initial model $\theta_{0}$, we adopt a simple method to find the language specific mask for each language pairs.

1. Start with a multilingual MT model $\theta_{0}$ jointly trained on $\left\{\mathcal{D}_{s_{i} \rightarrow t_{i}}\right\}_{i=1}^{N}$.

2. For each language pair $s_{i} \rightarrow t_{i}$, fine-tuning $\theta_{0}$ on $\mathcal{D}_{s_{i} \rightarrow t_{i}}$. Intuitively, fine-tuning $\theta_{0}$ on specific language pair $s_{i} \rightarrow t_{i}$ will amplify the magnitude of the important weights for $s_{i} \rightarrow t_{i}$ and diminish the magnitude of the unimportant weights.

3. Rank the weights in fine-tuned model and prune the lowest $\alpha$ percent. The mask $\mathbf{M}_{s_{i} \rightarrow t_{i}}$ is obtained by setting the remaining indices of parameters to be 1 .

\subsection{Structure-aware Joint Training}

Once we get masks $\mathbf{M}_{s_{i} \rightarrow t_{i}}$ for all language pairs, we further continue to train $\theta_{0}$ with languagegrouped batching and structure-aware updating.

First, we create random batches of bilingual sentence pairs where each batch contains only samples from one pair. This is different from the plain joint multilingual training where each batch can contain fully random sentence pairs from all languages Specifically, a batch $\mathcal{B}_{s_{i} \rightarrow t_{i}}$ is randomly drawn from the language-specific data $\mathcal{D}_{s_{i} \rightarrow t_{i}}$. Second, we evaluate the loss in Eq. 1 on the batch $\mathcal{B}_{s_{i} \rightarrow t_{i}}$. During the back-propagation step, we only update the parameters in $\theta_{0}$ belonging to the sub-network indicated by $\mathbf{M}_{s_{i} \rightarrow t_{i}}$. We iteratively update the parameters until convergence.
In this way, we still get a single final model $\theta^{*}$ that is able to translate all language directions.

During the inference, this model $\theta^{*}$ and its masks $\mathbf{M}_{s_{i} \rightarrow t_{i}}, i=1, \ldots, N$ are used together to make predictions. For every given input sentence in language $s$ and a target language $t$, the forward inference step only uses the parameter $\theta^{*} \odot \mathbf{M}_{s \rightarrow t}$ to calculate model output.

\section{Experiment Settings}

Datasets and Evaluation The experiments are conducted on IWSLT and WMT benchmarks. For IWSLT, we collect 8 English-centric language pairs from IWSLT2014, whose size ranges from $89 \mathrm{k}$ to $169 \mathrm{k}$. To simulate the scenarios of imbalanced datasets, we collect 18 language pairs ranging from low-resource $(\mathrm{Gu}, 11 \mathrm{k})$ to rich-resource $(\mathrm{Fr}, 37 \mathrm{~m})$ from previous years' WMT. The details of the datasets are listed in Appendix. We apply byte pair encoding (BPE) (Sennrich et al., 2016) to preprocess multilingual sentences, resulting in a vocabulary size of 30k for IWSLT and 64k for WMT. Besides, we apply over-sampling for IWSLT and WMT to balance the training data distribution with a temperature of $T=2$ and $T=5$ respectively. Similar to Lin et al. (2020), we divide the language pairs into 3 categories: low-resource $(<1 \mathrm{M})$, medium-resource $(>1 \mathrm{M}$ and $<10 \mathrm{M})$ and rich resource $(>10 \mathrm{M})$.

We perform many-to-many multilingual translation throughout this paper, and add special language tokens at both the source and the target side. In all our experiments, we evaluate our model with commonly used standard testsets. For zeroshot, where standard testsets (for example, $\mathrm{Fr} \rightarrow \mathrm{Zh}$ ) of some language pairs are not available, we use OPUS-100 (Zhang et al., 2020) testsets instead.

We report tokenized BLEU, as well as win ratio (WR), informing the proportion of language pairs we outperform the baseline. In zero-shot translation, we also report translation-language accuracy ${ }^{1}$, which is commonly used to measure the accuracy of translating into the right target language.

Model Settings Considering the diversity of dataset volume, we perform our experiments with variants of Transformer architecture. For IWSLT, we adopt a smaller Transformer (Transformersmall $^{2}$ (Wu et al., 2019)). For WMT, we adopt

\footnotetext{
${ }^{1}$ https://github.com/Mimino666/ langdetect

${ }^{2}$ Transformer-base with $d_{f f}=1024$ and $n_{\text {head }}=4$
} 


\begin{tabular}{rcccc}
\hline Lang & $\mathrm{Fa}$ & $\mathrm{Pl}$ & $\mathrm{Ar}$ & $\mathrm{He}$ \\
Size & $89 \mathrm{~K}$ & $128 \mathrm{k}$ & $140 \mathrm{~K}$ & $144 \mathrm{~K}$ \\
\hline Baseline & 16.9 & 16.4 & 20.9 & 29 \\
LaSS & $\mathbf{1 7 . 9}$ & $\mathbf{1 7 . 0}$ & $\mathbf{2 2 . 9}$ & $\mathbf{3 0 . 9}$ \\
$\Delta$ & +1.0 & +0.6 & +2.0 & +1.9 \\
\hline \hline Lang & $\mathrm{Nl}$ & $\mathrm{De}$ & $\mathrm{It}$ & $\mathrm{Es}$ \\
Size & $153 \mathrm{~K}$ & $160 \mathrm{~K}$ & $167 \mathrm{~K}$ & $169 \mathrm{~K}$ \\
\hline Baseline & 30.9 & 28.1 & 29.2 & 35.2 \\
LaSS & $\mathbf{3 3 . 0}$ & $\mathbf{2 9 . 8}$ & $\mathbf{3 0 . 9}$ & $\mathbf{3 7 . 3}$ \\
$\Delta$ & +2.1 & +1.7 & +1.7 & +2.1 \\
\hline
\end{tabular}

Table 1: Results on IWLST dataset. Baseline denotes the multilingual Transformer-small baseline model. LaSS consistently outperforms multilingual baseline on all language pairs. We report the average BLEU of $\mathrm{En} \rightarrow \mathrm{X}$ and $\mathrm{X} \rightarrow \mathrm{En}$ within one language. Both the baseline and LaSS have the same number of parameters.

Transformer-base and Transformer-big ${ }^{3}$. The pruning rate $\alpha$ of IWSLT and WMT is 0.7 and 0.3 , respectively. For simplicity, we only report the highest BLEU from the best pruning rate and we also discuss the impact of different pruning rate on performance in Sec.6. In Sec. 6 we discuss the relationship of performance and pruning rate. For more training details please refer to Appendix.

\section{Experiment Results}

This section shows the efficacy and generalization of LaSS. Firstly, we show that LaSS obtains consistent performance gains on IWSLT and WMT datasets with different Transformer architecture variants. Further, we show that LaSS can easily generalize to new language pairs without losing the accuracy for previous language pairs. Finally, we observe that LaSS can even improve zero-shot translation, obtaining performance gains by up to 26.5 BLEU.

\subsection{Main Results}

Results on IWSLT We first show our results on IWSLT. As shown in Table 1, LaSS consistently outperforms the multilingual baseline on all language pairs, confirming that using LaSS to alleviate parameter interference can help boost performance.

Results on WMT To further verify the generalization of LaSS, we also conduct experiments on

\footnotetext{
${ }^{3}$ For details of the Transformer setting, please refer to Vaswani et al. (2017)
}

WMT, where the dataset is more imbalanced across different language pairs. We adopt two different Transformer architecture variants, i.e., Transformerbase and Transformer-big.

As shown in Table 2, LaSS obtains consistent gains over multilingual baseline on WMT for both Transformer-base and Transformer-big. For Transformer-base, LaSS achieves an average improvement of 1.2 BLEU on 36 language pairs over baseline, while for Transformer-big, LaSS obtains 0.6 BLEU improvement.

We observe that with the dataset scale of language pairs increasing, the improvements of BLEU and WR become larger, suggesting that the language pairs with large scale dataset benefit more from LaSS than language pairs of low resource. This phenomenon is intuitive since rich resource dataset suffers more parameter interference than low resource dataset. We also find that the BLEU and WR gains obtained in Transformer-base are larger than that in Transformer-large. We attribute it to the more severe parameter interference for smaller models.

For comparison, we also include the results of LaSS with randomly initialized masks. Not surprising, Random underperforms the baseline by a large margin, since Random intensifies rather than alleviates the parameter interference.

\subsection{Generalization to New Language Pairs}

LaSS has shown its efficacy in the above section. A natural question arises that can LaSS adapt to a new language or language pair that it has not seen in training phase? In other words, can LaSS generalize to other language pairs? In this section, we show the generalization of LaSS in two settings. We firstly show that LaSS can easily adapt to new unseen languages to match bilingual models with training for only a few hundred steps while keeping the performance of the existing language pairs hardly dropping. Secondly, we show that LaSS can also boost performance in zero-shot translation scenario, obtaining performance gains by up to 26.5 BLEU.

The model is Transformer-big trained on WMT dataset. $\mathrm{En} \leftrightarrow \mathrm{Ar}$ and $\mathrm{En} \leftrightarrow \mathrm{It}$ are both unseen language pairs.

\subsubsection{Extensibility to New Languages}

Previous works have studied the easy and rapid adaptation to a new task or language pair (Bapna and Firat, 2019; Rebuffi et al., 2017). We show 


\begin{tabular}{lllllllllll}
\hline \multirow{2}{*}{ Arch Setting } & \multirow{2}{*}{ Model } & \multicolumn{2}{c}{ Low } & \multicolumn{2}{c}{ Medium } & \multicolumn{2}{c}{ Rich } & \multicolumn{2}{c}{ All } \\
& & BLEU & WR & BLEU & WR & BLEU & WR & BLEU & WR \\
\hline \multirow{3}{*}{ Transformer-base } & Baseline & 16.7 & - & 18.8 & - & 25.3 & - & 20.4 & \\
& Random & -2.2 & 0.0 & -2.3 & 0.0 & -2.6 & 0.0 & -2.4 & 0.0 \\
& LaSS & $\mathbf{+ 0 . 7}$ & $\mathbf{8 0 . 0}$ & $\mathbf{+ 1 . 3}$ & $\mathbf{8 5 . 7}$ & $\mathbf{+ 1 . 7}$ & $\mathbf{1 0 0 . 0}$ & $\mathbf{+ 1 . 2}$ & $\mathbf{8 8 . 9}$ \\
\hline \multirow{3}{*}{ Transformer-big } & Baseline & 18.8 & - & 22.2 & - & 29.0 & - & 23.5 & - \\
& Random & -1.3 & 0.0 & -1.8 & 0.0 & -1.5 & 0.0 & -1.6 & 0.0 \\
& LaSS & $\mathbf{+ 0 . 1}$ & $\mathbf{5 0 . 0}$ & $\mathbf{+ 0 . 7}$ & $\mathbf{9 2 . 9}$ & $\mathbf{+ 0 . 8}$ & $\mathbf{1 0 0 . 0}$ & $\mathbf{+ 0 . 6}$ & $\mathbf{8 3 . 3}$ \\
\hline
\end{tabular}

Table 2: Average BLEU $\uparrow$ and Win Ratio (WR) of WMT dataset on Low $(<1 \mathrm{M})$, Medium $(1 \mathrm{M} \sim 10 \mathrm{M})$ and Rich $(>10 \mathrm{M})$ resource dataset. Random denotes LaSS with random masks. LaSS obtains consistent gains for both Transformer-big and Transformer-base.

that LaSS can also easily adapt to new unseen languages without dramatic drop for other existing languages. We distribute a new sub-network to each new language pair and train the sub-network with the specific language pair for fixed steps. In this way, the new language pair will only update the corresponding parameters and it can alleviate the interference and catastrophic forgetting (Kirkpatrick et al., 2016) to other language pairs.

We verify the extensibility of LaSS on 4 language pairs. For LaSS, as described in Sec.3, we first fine-tune the multilingual base model and prune to obtain the specific mask for the new language pair. For both multilingual baseline and our method, we train on only the specific language pair for fixed steps.

Figure 2 shows the trend of BLEU score along with the training steps. We observe that 1) LaSS consistently outperforms the multilingual baseline model along with the training steps. LaSS reaches the bilingual model performance with fewer steps. 2) Besides, the degradation of other language pairs is much smoother than the baseline. When reaching the bilingual baseline performance, LaSS hardly drops on other language pairs, while the multilingual baseline model dramatically drops by a large margin.

We attribute the easy adaptation for specific languages to the language specific sub-network. LaSS only updates the corresponding parameters, avoiding updating all parameters which will hurt the performance of other languages. Another benefit of updating corresponding parameters is its fast adaptation towards specific language pairs.

\subsubsection{Zero-shot}

Zero-shot translation is the translation between known languages that the model has never seen

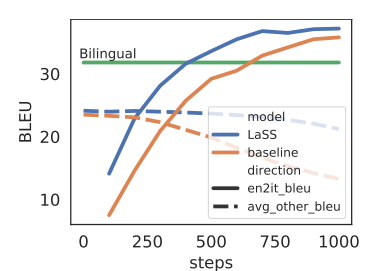

(a) $\mathrm{En} \rightarrow \mathrm{It}$

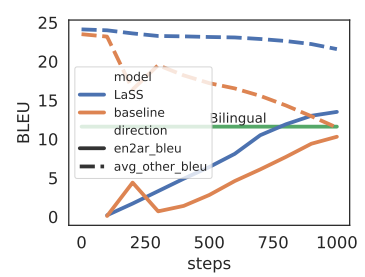

(c) $\mathrm{En} \rightarrow \mathrm{Ar}$

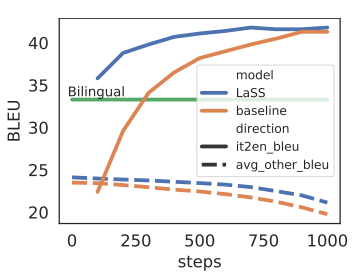

(b) It $\rightarrow$ En

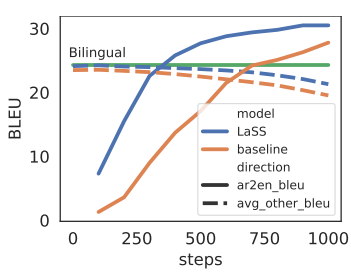

(d) $\mathrm{Ar} \rightarrow \mathrm{En}$
Figure 2: The trend of BLEU score of new extended language pairs and other existing language pairs along with the training steps on the specific language pair. Compared to multilingual baseline, LaSS reaches the bilingual performance with fewer steps and only little performance degradation on other existing language pairs.

together at training time (e.g., $\mathrm{Fr} \rightarrow \mathrm{En}$ and $\mathrm{En} \rightarrow \mathrm{Zh}$ are both seen in training phase, while $\mathrm{Fr} \rightarrow \mathrm{Zh}$ is not.). It is the ultimate goal of Multilingual NMT and has been a common indicator to measure the model capability (Johnson et al., 2017; Zhang et al., 2020). One of the biggest challenges is the offtarget issue (Zhang et al., 2020), which means that the model translates into a wrong target language.

In previous experiments, we apply specific masks to their corresponding language pairs. As the training dataset is English-centric, non-Englishcentric masks are not available. We remedy it by merging two masks to create non-English-centric masks. For example, We create $\mathrm{X} \rightarrow \mathrm{Y}$ mask by combining the encoder mask of $\mathrm{X} \rightarrow \mathrm{En}$ and the 

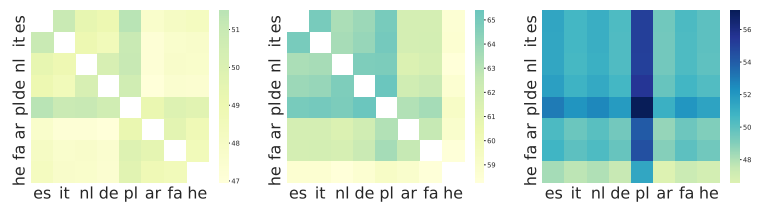

(a) En $\rightarrow \mathrm{X}$ ( $\mathrm{x}$-axis (b) $\mathrm{X} \rightarrow$ En ( $\mathrm{x}$-axis (c) $\mathrm{X} \rightarrow$ En ( $\mathrm{X}$-axis) and $\mathrm{y}$-axis) and $\mathrm{y}$-axis)

$\mathrm{En} \rightarrow \mathrm{X}(\mathrm{y}$-axis $)$

Figure 3: Mask similarity for language pairs within $\mathrm{En} \rightarrow \mathrm{X}$ ( $\mathrm{x}$-axis and $\mathrm{y}$-axis), within $\mathrm{X} \rightarrow$ En ( $\mathrm{x}$-axis and $\mathrm{y}-$ axis) and between $\mathrm{En} \rightarrow \mathrm{X}$ (X-axis) and $\mathrm{X} \rightarrow \mathrm{En}$ (y-axis), respectively. The mask similarity is positively correlated to the language family similarity.

decoder mask of $\mathrm{En} \rightarrow \mathrm{Y}$. We select 6 languages and evaluate zero-shot translation in language pairs between each other.

As shown in Table 3, surprisingly, by directly applying $\mathrm{X} \rightarrow \mathrm{Y}$ masks, LaSS obtains consistent gains over baselines in all language pairs for both BLEU and translation-language accuracy, indicating that the superiority of LaSS in learning to bridge between languages. It is worth noting that for $\mathrm{Fr} \rightarrow \mathrm{Zh}$, LaSS outperforms the baseline by 26.5 BLEU, reaching 32 BLEU.

We also sample a few translation examples from $\mathrm{Fr} \rightarrow \mathrm{Zh}$ to analyze why LaSS can help boost zeroshot (More examples are listed in Appendix).

As shown in Table 4 as well as translationlanguage accuracy in Table 3, we observe that the multilingual baseline has severe off-target issue. As a counterpart, LaSS significantly alleviates the off-target issue, translating into the right target language. We attribute the success of "on-target" in zero-shot to the language specific parameters as a strong signal, apart from language indicator, to the model to translate into the target language.

\section{Analysis and Discussion}

In this section, we conduct a set of analytic experiments to better understand the characteristics of language specific sub-network. We first measure the relationship between language specific subnetwork as well as its capacity and language family. Secondly, we study how masks affect performance in zero-shot scenario. Lastly, we discuss the relationship between pruning rate $\alpha$ and performance.

We conduct our analytic experiments on IWSLT dataset. For readers not familiar with language family and clustering, Figure 4 is the hierarchical clustering according to language family.

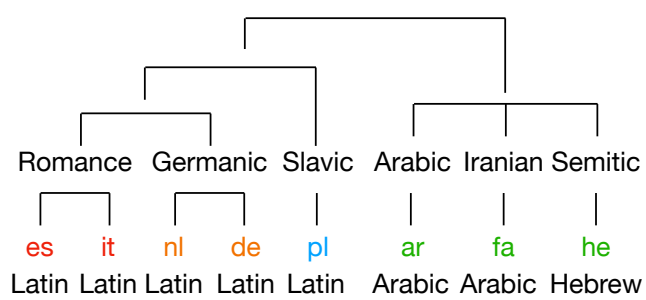

Figure 4: Language clustering of 8 languages in IWSLT, according to language family. Es(Spanish), It(Italian), De(Germany), N1(Dutch) and $\mathrm{Pl}$ (Polish) are all European languages and written in Latin while Ar(Arabic), $\mathrm{Fa}$ (Farsi) and $\mathrm{He}$ (Hebrew) are similar languages.

\subsection{Mask similarity v.s Language family}

Ideally, similar languages should share more parameters since they share more language characteristics. Therefore, a natural question arises: Does the model automatically capture the relationship of language family defined by human?

We calculate the similarity of masks between language pairs to measure the sub-network relationship between language pairs. We define mask similarity as the number of 1 where two masks share divided by the number of 1 of the first mask:

$$
\operatorname{Sim}\left(\mathbf{M}_{1}, \mathbf{M}_{2}\right)=\frac{\left\|\mathbf{M}_{1} \cap \mathbf{M}_{2}\right\|_{0}}{\left\|\mathbf{M}_{1}\right\|_{0}},
$$

where $\|\cdot\|_{0}$ represent $L_{0}$ norm. Mask similarity reflects the degree of sharing among different language pairs.

Figure 3(a) and 3(b) shows the mask similarity in $E n \rightarrow X$ and $X \rightarrow E n$. We observe that, for both $\mathrm{En} \rightarrow \mathrm{X}$ and $\mathrm{X} \rightarrow \mathrm{En}$, the mask similarity is positively correlated to the language family similarity. The color of grids in Figure is deeper between similar languages (for example, es and it) while more shallow between dissimilar languages (for example, es and he).

We also plot the similarity between $\mathrm{En} \rightarrow \mathrm{X}$ and $\mathrm{X} \rightarrow$ En in Figure 3(c). We observe that, unlike $\mathrm{En} \rightarrow \mathrm{X}$ or $\mathrm{X} \rightarrow \mathrm{En}$, the mask similarity does not correspond to language family similarity. We suspect that the mask similarity is determined by combination of source and target languages. That means that $\mathrm{En} \rightarrow \mathrm{Nl}$ does not necessarily share more parameters with $\mathrm{Nl} \rightarrow$ En than $\mathrm{En} \rightarrow$ De.

\subsection{Where language specific capacity matters?}

To take a step further, we study how model schedule language specific capacity across layers. Figure 5 


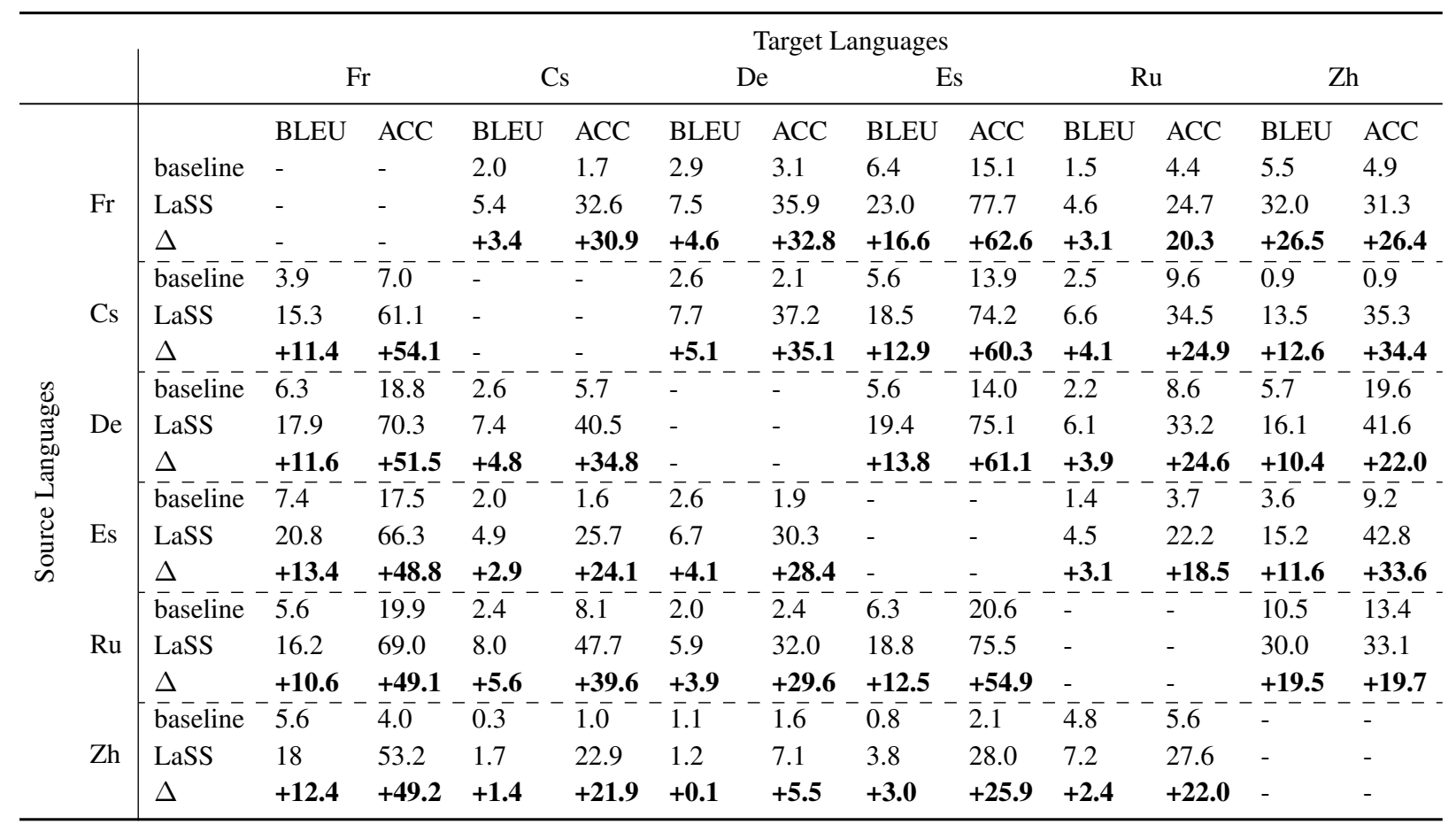

Table 3: BLEU score and Translation-language Accuracy (ACC, in percentage) of zero-shot translation for multilingual baseline and LaSS. LaSS outperforms the multilingual baseline on both BLEU and ACC by a large margin for most language pairs. Low accuracy indicates severe off-target translation.

\begin{tabular}{|c|c|}
\hline Src & $\begin{array}{l}\text { La production annuelle d'acier é tait le sym- } \\
\text { bole incontest é de la vigueur é conomique } \\
\text { des nations. }\end{array}$ \\
\hline & 钢的年产量是国家经济实力的重要象征 \\
\hline Baseline & $\begin{array}{l}\text { Annual steel production was the undisputed } \\
\text { symbol of nations' economic strength. }\end{array}$ \\
\hline LaSS & $\begin{array}{l}\text { 年度钢铁生产是各国经济活力的无可争 } \\
\text { 辩的象征. }\end{array}$ \\
\hline Src & $\begin{array}{l}\text { De l'avis de ma d é lé gation donc, l'ONU } \\
\text { devrait é largir ces activit é s de la faon suiv- } \\
\text { ante. }\end{array}$ \\
\hline Ref & $\begin{array}{l}\text { 因此,我国代表团认为,联合国现在应该 } \\
\text { 以下述方式扩大这些活动。 }\end{array}$ \\
\hline Baseline & $\begin{array}{l}\text { 因此, in my delegation's view, the United } \\
\text { Nations should expand these activities in the } \\
\text { following manner. }\end{array}$ \\
\hline LaSS & $\begin{array}{l}\text { 因此,我国代表团认为,联合国应该扩大 } \\
\text { 这些活动,如下. }\end{array}$ \\
\hline
\end{tabular}

Table 4: Fr $\rightarrow$ Zh Case Study. The multilingual baseline suffers from severe off-target issue, while LaSS greatly alleviates the issue.

shows the similarity of different components on the encoder and decoder side along with the increase of layer. More concretely, we plot query, key, value on the attention sub-layer and fully-connected layer on the positional-wise feed-forward sub-layer.

We observe that a) On both the encoder and decoder side, the model tends to distribute more language specific components on the top and bot- tom layers rather than the middle ones. This phenomenon is intuitive. The bottom layers deal more with embedding, which is language specific, while the top layers are near the output layer, which is also language specific. b) For fully-connected layer, the model tends to distribute more language specific capacity on the middle layers for the encoder, while distribute more language specific capacity in the decoder for the top layers.

\subsection{How masks affect zero-shot?}

In Sec.4, we show that simply applying $\mathrm{X} \rightarrow \mathrm{Y}$ masks can boost zero-shot performance. We conduct experiments to analyze how masks affect zeroperformance. Concretely, we take $\mathrm{Fr} \rightarrow \mathrm{Zh}$ as an example, replacing the encoder or decoder mask with another language mask, respectively.

As shown in Table 5, we observe that replacing the encoder mask with other languages causes only littler performance drop, while replacing the decoder mask causes dramatic performance drop. It suggests that the decoder mask is the key ingredient of performance improvement.

\subsection{About Sparsity}

To better understand the pruning rate, we plot the performance along with the increase of pruning 


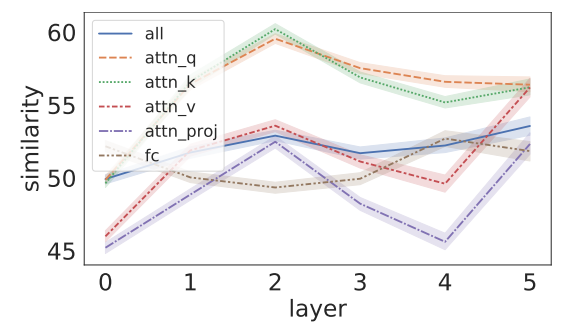

(a) Encoder

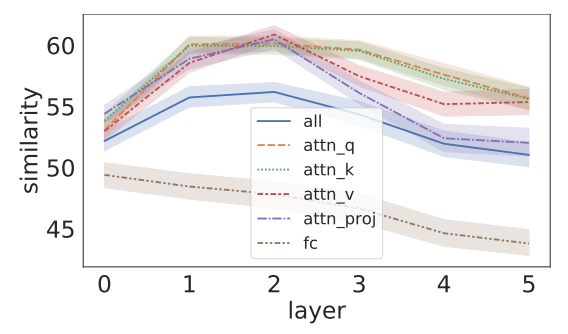

(b) Decoder

Figure 5: The mask similarity of different components (attention layer and feed-forward layer) on the encoder and decoder side along with the increase of layer. The model tends to distribute more language specific capacity on the top and bottom layers.

\begin{tabular}{llllll}
\hline \multicolumn{7}{c}{$\mathbf{F r} \rightarrow \mathbf{X}$} \\
\hline Fr & Cs & De & Es & Ru & Zh \\
- & 12.3 & 13.8 & 7.1 & 18.6 & $\mathbf{3 2 . 0}$ \\
\hline \hline \multicolumn{7}{c}{$\mathbf{X} \rightarrow \mathbf{Z h}$} \\
\hline Fr & Cs & De & Es & Ru & Zh \\
$\mathbf{3 2 . 0}$ & 30.5 & 29.6 & 30.9 & 29.6 & - \\
\hline
\end{tabular}

Table 5: Performance of applying $\mathrm{Fr} \rightarrow \mathrm{X}$ or $\mathrm{X} \rightarrow \mathrm{Zh}$ mask to $\mathrm{Fr} \rightarrow \mathrm{Zh}$ testset. Replacing encoder mask causes only little performance drop, while replacing decoder mask causes dramatic performance drop.

rate in Figure 6. For WMT, the best choice for $\alpha$ is 0.3 for both Transformer-base and Transformer-big, while for IWSLT the best $\alpha$ lies between 0.6 0.7 The results are consistent with our intuition, that large scale training data need a smaller pruning rate to keep the model capacity. Therefore, we suggest tuning $\alpha$ based on both the dataset and model size. For large datasets such as WMT, setting a smaller $\alpha$ is better, while a larger $\alpha$ will slightly decrease the performance (i.e. less than 0.5 BLEU score). For small datasets like IWSLT, setting a larger $\alpha$ may yield better performance.

\section{Conclusion}

In this paper, we propose to learn LanguageSpecific Sub-network (LaSS) for multilingual

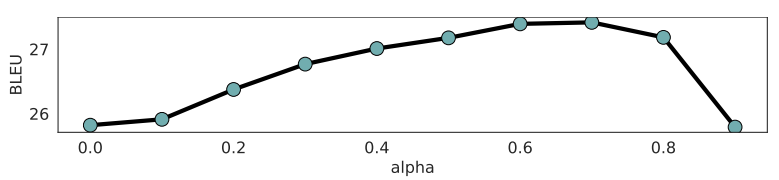

(a) IWSLT

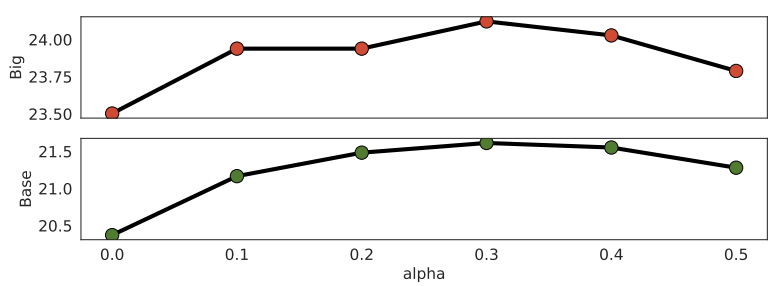

(b) WMT

Figure 6: BLEU score along with the increase of pruning rate $\alpha$. Large $\alpha$ indicates small sub-network. Small dataset requires a larger $\alpha$ to yield better performance. IWSLT uses Transformer-small and WMT uses Transformer-base and Transformer-big.

NMT. Extensive experiments on IWSLT and WMT have shown that LaSS is able to alleviate parameter interference and boost performance. Further, LaSS can generalize well to new language pairs by training with a few hundred steps, while keeping the performance of existing language pairs. Surprisingly, in zero-shot translation, LaSS surpasses the multilingual baseline by up to 26.5 BLEU. Extensive analytic experiments are conducted to understand the characteristics of language specific sub-network. Future work includes designing a more dedicated end-to-end training strategy and incorporating the insight we gain from analysis to design a further improved LaSS.

\section{References}

Roee Aharoni, Melvin Johnson, and Orhan Firat. 2019. Massively multilingual neural machine translation. In Proceedings of the 2019 Conference of the North American Chapter of the Association for Computational Linguistics: Human Language Technologies, NAACL-HLT 2019, Minneapolis, MN, USA, June 27, 2019, Volume 1 (Long and Short Papers), pages 3874-3884. Association for Computational Linguistics.

Naveen Arivazhagan, Ankur Bapna, Orhan Firat, Dmitry Lepikhin, Melvin Johnson, Maxim Krikun, Mia Xu Chen, Yuan Cao, George F. Foster, Colin Cherry, Wolfgang Macherey, Zhifeng Chen, and Yonghui Wu. 2019. Massively multilingual neural machine translation in the wild: Findings and challenges. CoRR, abs/1907.05019.

Dzmitry Bahdanau, Kyunghyun Cho, and Yoshua Bengio. 2015. Neural machine translation by jointly 
learning to align and translate. In 3rd International Conference on Learning Representations, ICLR 2015, San Diego, CA, USA, May 7-9, 2015, Conference Track Proceedings.

Ankur Bapna and Orhan Firat. 2019. Simple, scalable adaptation for neural machine translation. In Proceedings of the 2019 Conference on Empirical Methods in Natural Language Processing and the 9th International Joint Conference on Natural Language Processing, EMNLP-IJCNLP 2019, Hong Kong, China, November 3-7, 2019, pages 15381548. Association for Computational Linguistics.

Daxiang Dong, Hua Wu, Wei He, Dianhai Yu, and Haifeng Wang. 2015. Multi-task learning for multiple language translation. In Proceedings of the 53rd Annual Meeting of the Association for Computational Linguistics and the 7th International Joint Conference on Natural Language Processing of the Asian Federation of Natural Language Processing, ACL 2015, July 26-31, 2015, Beijing, China, Volume 1: Long Papers, pages 1723-1732. The Association for Computer Linguistics.

Utku Evci, Trevor Gale, Jacob Menick, Pablo Samuel Castro, and Erich Elsen. 2020. Rigging the lottery: Making all tickets winners. In Proceedings of the 37th International Conference on Machine Learning, ICML 2020, 13-18 July 2020, Virtual Event, volume 119 of Proceedings of Machine Learning Research, pages 2943-2952. PMLR.

Angela Fan, Shruti Bhosale, Holger Schwenk, Zhiyi Ma, Ahmed El-Kishky, Siddharth Goyal, Mandeep Baines, Onur Celebi, Guillaume Wenzek, Vishrav Chaudhary, Naman Goyal, Tom Birch, Vitaliy Liptchinsky, Sergey Edunov, Edouard Grave, Michael Auli, and Armand Joulin. 2020. Beyond english-centric multilingual machine translation. CoRR, abs/2010.11125.

Orhan Firat, Kyunghyun Cho, and Yoshua Bengio. 2016. Multi-way, multilingual neural machine translation with a shared attention mechanism. In NAACL HLT 2016, The 2016 Conference of the North American Chapter of the Association for Computational Linguistics: Human Language Technologies, San Diego California, USA, June 12-17, 2016, pages 866-875. The Association for Computational Linguistics.

Jonathan Frankle and Michael Carbin. 2019. The lottery ticket hypothesis: Finding sparse, trainable neural networks. In 7th International Conference on Learning Representations, ICLR 2019, New Orleans, LA, USA, May 6-9, 2019. OpenReview.net.

Hany Hassan, Anthony Aue, Chang Chen, Vishal Chowdhary, Jonathan Clark, Christian Federmann, Xuedong Huang, Marcin Junczys-Dowmunt, William Lewis, Mu Li, Shujie Liu, Tie-Yan Liu, Renqian Luo, Arul Menezes, Tao Qin, Frank Seide, Xu Tan, Fei Tian, Lijun Wu, Shuangzhi Wu, Yingce Xia, Dongdong Zhang, Zhirui Zhang, and Ming
Zhou. 2018. Achieving human parity on automatic chinese to english news translation. CoRR, abs/1803.05567.

Melvin Johnson, Mike Schuster, Quoc V. Le, Maxim Krikun, Yonghui Wu, Zhifeng Chen, Nikhil Thorat, Fernanda Viégas, Martin Wattenberg, Greg Corrado, Macduff Hughes, and Jeffrey Dean. 2017. Google's multilingual neural machine translation system: Enabling zero-shot translation. Transactions of the Association for Computational Linguistics, 5:339-351.

James Kirkpatrick, Razvan Pascanu, Neil C. Rabinowitz, Joel Veness, Guillaume Desjardins, Andrei A. Rusu, Kieran Milan, John Quan, Tiago Ramalho, Agnieszka Grabska-Barwinska, Demis Hassabis, Claudia Clopath, Dharshan Kumaran, and Raia Hadsell. 2016. Overcoming catastrophic forgetting in neural networks. CoRR, abs/1612.00796.

Taku Kudo and John Richardson. 2018. Sentencepiece: A simple and language independent subword tokenizer and detokenizer for neural text processing. In Proceedings of the 2018 Conference on Empirical Methods in Natural Language Processing, EMNLP 2018: System Demonstrations, Brussels, Belgium, October 31 - November 4, 2018, pages 66-71. Association for Computational Linguistics.

Xian Li, Asa Cooper Stickland, Yuqing Tang, and Xiang Kong. 2020. Deep transformers with latent depth. In Advances in Neural Information Processing Systems 33: Annual Conference on Neural Information Processing Systems 2020, NeurIPS 2020, December 6-12, 2020, virtual.

Zehui Lin, Xiao Pan, Mingxuan Wang, Xipeng Qiu, Jiangtao Feng, Hao Zhou, and Lei Li. 2020. Pretraining multilingual neural machine translation by leveraging alignment information. In Proceedings of the 2020 Conference on Empirical Methods in Natural Language Processing, EMNLP 2020, Online, November 16-20, 2020, pages 2649-2663. Association for Computational Linguistics.

Yinhan Liu, Jiatao Gu, Naman Goyal, Xian Li, Sergey Edunov, Marjan Ghazvininejad, Mike Lewis, and Luke Zettlemoyer. 2020. Multilingual denoising pre-training for neural machine translation. CoRR, abs/2001.08210

Zhuang Liu, Mingjie Sun, Tinghui Zhou, Gao Huang, and Trevor Darrell. 2019. Rethinking the value of network pruning. In 7th International Conference on Learning Representations, ICLR 2019, New Orleans, LA, USA, May 6-9, 2019. OpenReview.net.

Sungwon Lyu, Bokyung Son, Kichang Yang, and Jaekyoung Bae. 2020. Revisiting modularized multilingual NMT to meet industrial demands. In Proceedings of the 2020 Conference on Empirical Methods in Natural Language Processing, EMNLP 2020, Online, November 16-20, 2020, pages 5905-5918. Association for Computational Linguistics. 
Jerin Philip, Alexandre Berard, Matthias Gallé, and Laurent Besacier. 2020. Monolingual adapters for zero-shot neural machine translation. In Proceedings of the 2020 Conference on Empirical Methods in Natural Language Processing, EMNLP 2020, Online, November 16-20, 2020, pages 4465-4470. Association for Computational Linguistics.

Sylvestre-Alvise Rebuffi, Hakan Bilen, and Andrea Vedaldi. 2017. Learning multiple visual domains with residual adapters. In Advances in Neural Information Processing Systems 30: Annual Conference on Neural Information Processing Systems 2017, December 4-9, 2017, Long Beach, CA, USA, pages 506-516.

Devendra Singh Sachan and Graham Neubig. 2018. Parameter sharing methods for multilingual selfattentional translation models. In Proceedings of the Third Conference on Machine Translation: Research Papers, WMT 2018, Belgium, Brussels, October 31 November 1, 2018, pages 261-271. Association for Computational Linguistics.

Rico Sennrich, Barry Haddow, and Alexandra Birch 2016. Neural machine translation of rare words with subword units. In Proceedings of the 54th Annual Meeting of the Association for Computational Linguistics, ACL 2016, August 7-12, 2016, Berlin, Germany, Volume 1: Long Papers. The Association for Computer Linguistics.

Aditya Siddhant, Ankur Bapna, Yuan Cao, Orhan Firat, Mia Xu Chen, Sneha Reddy Kudugunta, Naveen Arivazhagan, and Yonghui Wu. 2020. Leveraging monolingual data with self-supervision for multilingual neural machine translation. In Proceedings of the 58th Annual Meeting of the Association for Computational Linguistics, ACL 2020, Online, July 5-10, 2020, pages 2827-2835. Association for Computational Linguistics.

Jinsong Su, Shan Wu, Deyi Xiong, Yaojie Lu, Xianpei Han, and Biao Zhang. 2018. Variational recurrent neural machine translation. In Proceedings of the Thirty-Second AAAI Conference on Artificial Intelligence, (AAAI-18), the 30th innovative Applications of Artificial Intelligence (IAAI-18), and the 8th AAAI Symposium on Educational Advances in Artificial Intelligence (EAAI-18), New Orleans, Louisiana, USA, February 2-7, 2018, pages 5488-5495. AAAI Press.

Tianxiang Sun, Yunfan Shao, Xiaonan Li, Pengfei Liu, Hang Yan, Xipeng Qiu, and Xuanjing Huang. 2020. Learning sparse sharing architectures for multiple tasks. In The Thirty-Fourth AAAI Conference on Artificial Intelligence, AAAI 2020, The ThirtySecond Innovative Applications of Artificial Intelligence Conference, IAAI 2020, The Tenth AAAI Symposium on Educational Advances in Artificial Intelligence, EAAI 2020, New York, NY, USA, February 7-12, 2020, pages 8936-8943.

Xu Tan, Yi Ren, Di He, Tao Qin, Zhou Zhao, and Tie-Yan Liu. 2019. Multilingual neural machine translation with knowledge distillation. In 7th International Conference on Learning Representations, ICLR 2019, New Orleans, LA, USA, May 6-9, 2019.

Ashish Vaswani, Noam Shazeer, Niki Parmar, Jakob Uszkoreit, Llion Jones, Aidan N. Gomez, Lukasz Kaiser, and Illia Polosukhin. 2017. Attention is all you need. In Advances in Neural Information Processing Systems 30: Annual Conference on Neural Information Processing Systems 2017, 4-9 December 2017, Long Beach, CA, USA, pages 5998-6008.

Mingxuan Wang. 2019. Towards linear time neural machine translation with capsule networks. In Proceedings of the 2019 Conference on Empirical Methods in Natural Language Processing and the 9th International Joint Conference on Natural Language Processing, EMNLP-IJCNLP 2019, Hong Kong, China, November 3-7, 2019, pages 803-812. Association for Computational Linguistics.

Qiang Wang, Bei Li, Tong Xiao, Jingbo Zhu, Changliang Li, Derek F. Wong, and Lidia S. Chao. 2019. Learning deep transformer models for machine translation. In Proceedings of the 57th Conference of the Association for Computational Linguistics, ACL 2019, Florence, Italy, July 28- August 2, 2019, Volume 1: Long Papers, pages 1810-1822. Association for Computational Linguistics.

Yining Wang, Jiajun Zhang, Feifei Zhai, Jingfang Xu, and Chengqing Zong. 2018. Three strategies to improve one-to-many multilingual translation. In Proceedings of the 2018 Conference on Empirical Methods in Natural Language Processing, Brussels, Belgium, October 31 - November 4, 2018, pages 29552960. Association for Computational Linguistics.

Felix Wu, Angela Fan, Alexei Baevski, Yann N. Dauphin, and Michael Auli. 2019. Pay less attention with lightweight and dynamic convolutions. In 7th International Conference on Learning Representations, ICLR 2019, New Orleans, LA, USA, May 69, 2019. OpenReview.net.

Yonghui Wu, Mike Schuster, Zhifeng Chen, Quoc V. Le, Mohammad Norouzi, Wolfgang Macherey, Maxim Krikun, Yuan Cao, Qin Gao, Klaus Macherey, Jeff Klingner, Apurva Shah, Melvin Johnson, Xiaobing Liu, Lukasz Kaiser, Stephan Gouws, Yoshikiyo Kato, Taku Kudo, Hideto Kazawa, Keith Stevens, George Kurian, Nishant Patil, Wei Wang, Cliff Young, Jason Smith, Jason Riesa, Alex Rudnick, Oriol Vinyals, Greg Corrado, Macduff Hughes, and Jeffrey Dean. 2016. Google's neural machine translation system: Bridging the gap between human and machine translation. CoRR, abs/1609.08144.

Biao Zhang, Ankur Bapna, Rico Sennrich, and Orhan Firat. 2021. Share or not? learning to schedule language-specific capacity for multilingual translation. In International Conference on Learning Representations. 
Biao Zhang, Philip Williams, Ivan Titov, and Rico Sennrich. 2020. Improving massively multilingual neural machine translation and zero-shot translation. In Proceedings of the 58th Annual Meeting of the Association for Computational Linguistics, pages 1628 1639, Online. Association for Computational Linguistics.

Michael Zhu and Suyog Gupta. 2018. To prune, or not to prune: Exploring the efficacy of pruning for model compression. In 6th International Conference on Learning Representations, ICLR 2018, Vancouver, BC, Canada, April 30 - May 3, 2018, Workshop Track Proceedings.

\section{A Appendices}

\section{A.1 Datasets Details}

\begin{tabular}{lllll}
\hline ISO & Language & Family & Script & Size \\
\hline fa & Farsi & Iranian & Arabic & $89 \mathrm{k}$ \\
ar & Arabic & Arabic & Arabic & $140 \mathrm{k}$ \\
he & Hebrew & Semitic & Hebrew & $144 \mathrm{k}$ \\
\hline nl & Dutch & Germanic & Latin & $153 \mathrm{k}$ \\
de & German & Germanic & Latin & $160 \mathrm{k}$ \\
\hline it & Italian & Romance & Latin & $167 \mathrm{k}$ \\
es & Spanish & Romance & Latin & $169 \mathrm{k}$ \\
\hline pl & Polish & Slavic & Latin & $128 \mathrm{k}$ \\
\hline
\end{tabular}

Table 6: Statistics and Language Family of IWSLT. Languages grouped together are similar languages.

\section{A.2 Training Details}

As stated in the previous section, we first train a multilingual baseline (Phase 1). Then we fine-tune the baseline on specific language pair to obtain the mask (Phase 2). After that we train the LaSS model with the obtained masks (Phase 3). Note that we only apply masks on linear weights, which means that the embedding weights, layer normalization are not masked out. We also exclude the output projection weight. We apply label smoothing of value 0.1 in all our experiments.

\section{A.2.1 IWSLT}

Model We adopt Transformer-small ${ }^{4}$ with dropout 0.1 .

Data Following Tan et al. (2019), we first tokenize the data then apply BPE. The BPE vocab size is $30 \mathrm{k}$. We apply over-sampling with a temperature of $T=2$.

Training For Phase 1, we train the baseline with Adam with a learning rate schedule of $(5 \mathrm{e}-4,4 \mathrm{k})$. The max tokens per batch is set to 262144. For Phase 2, we keep all other settings unchanged except we set the max tokens to be 16384 and the dropout 0.3. For Phase 3, we keep the same setting as Phase 1, except we apply masks on the model.

\section{A.2.2 WMT}

Model We adopt Transformer-base and Transformer-big with pre-norm (Wang et al.,

\footnotetext{
${ }^{4}$ Transformer-base with $d_{f f}=1024$ and $n_{\text {head }}=4$
} 


\begin{tabular}{lllllllc}
\hline ISO & Language & Family & Script & Train & Valid & Test & Size \\
\hline gu & Gujarati & Indo-Aryan & Gujarati & WMT19 & newsdev19 & newstest19 & $11 \mathrm{k}$ \\
ta & Tamil & Dravidian & Tamil & WMT20 & newsdev20 & newstest20 & $64 \mathrm{k}$ \\
\hline $\mathrm{kk}$ & Kazakh & Turkic & Cyrillic & WMT19 & newsdev19 & newstest19 & $120 \mathrm{k}$ \\
tr & Turkish & Turkic & Latin & WMT16 & newsdev16 & newstest16 & 205k \\
\hline ro & Romanian & Romance & Latin & WMT16 & newsdev16 & newstest16 & 597k \\
es & Spanish & Romance & Latin & WMT13 & newstest12 & newstest13 & $13 \mathrm{~m}$ \\
fr & French & Romance & Latin & WMT14 & newstest13 & newstest14 & $37 \mathrm{~m}$ \\
\hline ps & Pashto & Iranian & Arabic & WMT20 & newsdev20 & newstest20 & $1 \mathrm{~m}$ \\
\hline fi & Farsi & Uralic & Latin & WMT16 & newstest15 & newstest16 & $2 \mathrm{~m}$ \\
lv & Latvian & Baltic & Latin & WMT17 & newsdev17 & newstest17 & $2 \mathrm{~m}$ \\
et & Estonian & Uralic & Latin & WMT18 & newsdev18 & newstest18 & $2.1 \mathrm{~m}$ \\
\hline lt & Lithuanian & Baltic & Latin & WMT19 & newsdev19 & newstest19 & $2.3 \mathrm{~m}$ \\
\hline ru & Russian & Slavic & Cyrillic & WMT16 & newstest15 & newstest16 & $2.5 \mathrm{~m}$ \\
\hline cs & Czech & Slavic & Latin & WMT14 & newstest13 & newstest14 & $11 \mathrm{~m}$ \\
$\mathrm{pl}$ & Polish & Slavic & Latin & WMT20 & newsdev20 & newstest20 & $11.1 \mathrm{~m}$ \\
\hline ja & Japanese & Japonic & Kanji; Kana & WMT20 & newsdev20 & newstest20 & $16.8 \mathrm{~m}$ \\
zh & Chinese & Chinese & Chinese & WMT17 & newsdev17 & newstest17 & $20.8 \mathrm{~m}$ \\
\hline de & German & Germanic & Latin & WMT16 & newstest13 & newstest14 & $4.5 \mathrm{~m}$ \\
\hline
\end{tabular}

Table 7: Statistics and Language Family of WMT daatset. Languages grouped together are similar languages.

\begin{tabular}{|c|c|c|c|c|c|c|c|}
\hline & \multicolumn{6}{|c|}{ Tgt } \\
\hline & & $\mathrm{Fr}$ & $\mathrm{Cs}$ & De & Es & $\mathrm{Ru}$ & $\mathrm{Zh}$ \\
\hline \multirow{6}{*}{ 号 } & $\mathrm{Fr}$ & & nt13 & nt13 & nt13 & $\mathrm{nt} 13$ & opus \\
\hline & $\mathrm{Cs}$ & & & nt13 & nt13 & $\mathrm{nt} 13$ & ted \\
\hline & $\mathrm{De}$ & & & & nt13 & nt13 & opus \\
\hline & Es & & & & & nt13 & ted \\
\hline & $\mathrm{Ru}$ & & & & & & opus \\
\hline & $\mathrm{Zh}$ & & & & & & \\
\hline
\end{tabular}

Table 8: Datasets used in Zero-shot Translation. "nt13" indicates newstest2013.

2019). We replace fixed positional embedding with learnable one and replace ReLU with GeLU. Also we use Layernorm-embedding (Liu et al., 2020) to stabilize training.

Data We use SentencePiece (Kudo and Richardson, 2018) to preprocess the data and learn BPE. Since the WMT dataset is highly imbalanced, we apply a temperature-based sampling strategy with $T=5$. To ensure all languages are represented adequately in the vocabulary, we apply the same temperature-based sampling strategy for training the BPE model.

Training For Phase 1, we train the baseline with Adam with a learning rate schedule of $(5 \mathrm{e}-4,8 \mathrm{k})$. The max tokens per batch is set to 524288. For Phase 2, the warm-up updates are set to 1000 . To guarantee that the model does not overfit the data, we train on different language pairs with different steps and different batch size. Concretely, we finetune on $>10 \mathrm{k},>100 \mathrm{k},>1 \mathrm{~m},>10 \mathrm{~m}$ language pairs with $1 \mathrm{k}, 2 \mathrm{k}, 4 \mathrm{k}, 8 \mathrm{k}$ steps and max tokens per batch with 20480, 40960, 81920 and 163840. For Phase 3 , we keep the setting the same as Phase 1 .

\section{A.3 Case Study}




\begin{tabular}{|c|c|}
\hline \multicolumn{2}{|r|}{$\mathbf{F r} \rightarrow \mathbf{Z h}$} \\
\hline Src & $\begin{array}{l}\text { La production annuelle d'acier é tait le symbole incontest é de la vigueur } \\
\text { é conomique des nations. }\end{array}$ \\
\hline Ref & 钢的年产量是国家经济实力的重要象征 \\
\hline $\begin{array}{l}\text { Baseline } \\
\text { LaSS }\end{array}$ & $\begin{array}{l}\text { Annual steel production was the undisputed symbol of nations' economic strength. } \\
\text { 年度钢铁生产是各国经济活力的无可争辩的象征. }\end{array}$ \\
\hline Src & $\begin{array}{l}\text { De l'avis de ma d é lé gation donc, l'ONU devrait é largir ces activit é s de la faon } \\
\text { suivante. }\end{array}$ \\
\hline Ref & 因此,我国代表团认为,联合国现在应该以下述方式扩大这些活动。 \\
\hline Baseline & $\begin{array}{l}\text { 因此, in my delegation's view, the United Nations should expand these activities in } \\
\text { the following manner. }\end{array}$ \\
\hline LaSS & 因此,我国代表团认为,联合国应该扩大这些活动,如下. \\
\hline & $\begin{array}{l}\text { Le domicile de la femme d é pendait du lieu du mariage et de la r é sidence familiale. } \\
\text { 妇女的住处取决于婚姻和家庭位置。 }\end{array}$ \\
\hline Baseline & $\begin{array}{l}\text { The woman's place of residence depended on the place of marriage and family } \\
\text { residence. }\end{array}$ \\
\hline LaSS & 妻子的住所取决于婚姻地点和家庭住所. \\
\hline \multicolumn{2}{|r|}{$\mathbf{D e} \rightarrow \mathbf{Z h}$} \\
\hline Src & Du bist gebissen worden. \\
\hline Ref & 你被咬了 \\
\hline $\begin{array}{l}\text { Baseline } \\
\text { LaSS }\end{array}$ & $\begin{array}{l}\text { You have been bitten. } \\
\text { 你被咬了 }\end{array}$ \\
\hline Src & Einmal w ü rde schon reichen. \\
\hline Ref & 你只需要道歉一次就够了! \\
\hline $\begin{array}{l}\text { Baseline } \\
\text { LaSS }\end{array}$ & $\begin{array}{l}\text { Once upon a time it would be enough. } \\
\text { 一次就足够了. }\end{array}$ \\
\hline $\begin{array}{l}\text { Src } \\
\text { Ref }\end{array}$ & $\begin{array}{l}\text { Wenn wir warten, hat er Zeit zum Tanken und Munitionieren. } \\
\text { 如果我们等待,他就有了 时间加油和补给弹药 }\end{array}$ \\
\hline $\begin{array}{l}\text { Baseline } \\
\text { LaSS }\end{array}$ & $\begin{array}{l}\text { When we wait, he has time for tanks and ammunition. } \\
\text { 当我们等待时,他有时间去坦克和弹药. }\end{array}$ \\
\hline \multicolumn{2}{|r|}{$\mathbf{R u} \rightarrow \mathbf{Z h}$} \\
\hline Src & 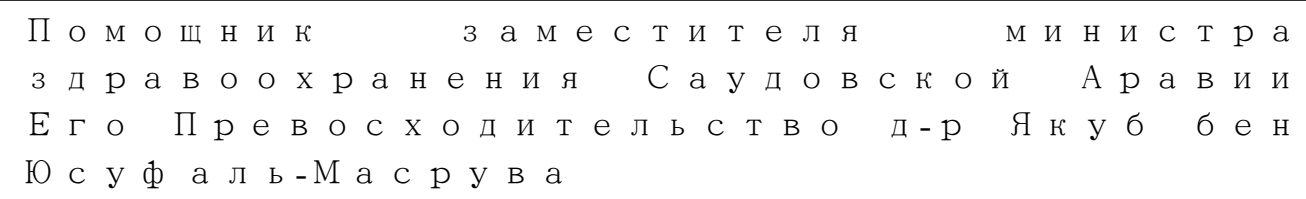 \\
\hline Ref & 沙特阿拉伯卫生部助理副部长雅各布 · 本 ·优素福 ·马斯如瓦博士阁下 \\
\hline $\begin{array}{l}\text { Baseline } \\
\text { LaSS }\end{array}$ & $\begin{array}{l}\text { Dr Yakub bin Yusuf al-Masruva, Deputy Minister of Health of Saudi Arabia } \\
\text { 沙特阿拉伯卫生部副部长的助理,His Excellency Dr Yakub bin Yusuf al-Masruva }\end{array}$ \\
\hline Src & 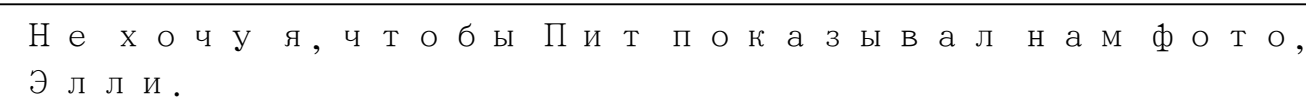 \\
\hline Ref & 我不要皮特给我们看照片 艾莉 \\
\hline Baseline & I don't want Pete showing us a photo, Elly. \\
\hline LaSS & 我不想让皮特给我们看一下照片,艾丽. \\
\hline Src & Р о дж е р с ! Я с к а з а л, в с т а т ь в \\
\hline Ref & 罗杰斯 我说跟上 \\
\hline Baseline & 罗吉尔斯! I said, get up! \\
\hline LaSS & 罗吉尔斯,我说,你要站起来! \\
\hline
\end{tabular}

